\title{
Ultrasound Servoing of Catheters for Beating Heart Valve Repair
}

\section{Citation}

Kesner, Samuel B., Shelten G. Yuen, and Robert D. Howe. 2010. Ultrasound servoing of catheters for beating heart valve repair. In Information Processing in Computer-Assisted Interventions, ed. N. Navab, Nassir and P. Jannin. Springer. Previously published in Lecture Notes in Computer Science 6135.

\section{Published Version}

http://www.springer.com/computer/hci/book/978-3-642-13710-5

\section{Permanent link}

http://nrs.harvard.edu/urn-3:HUL.InstRepos:4262333

\section{Terms of Use}

This article was downloaded from Harvard University's DASH repository, and is made available under the terms and conditions applicable to Open Access Policy Articles, as set forth at http:// nrs.harvard.edu/urn-3:HUL.InstRepos:dash.current.terms-of-use\#OAP

\section{Share Your Story}

The Harvard community has made this article openly available.

Please share how this access benefits you. Submit a story.

\section{Accessibility}




\title{
Ultrasound Servoing of Catheters for Beating Heart Valve Repair
}

\author{
Samuel B. Kesner ${ }^{1}$, Shelten G. Yuen ${ }^{1}$, and Robert D. Howe ${ }^{1,2}$ \\ ${ }^{1}$ Harvard School of Engineering and Applied Sciences, Cambridge, MA \\ ${ }^{2}$ Harvard-MIT Division of Health Sciences \& Technology, Cambridge, MA
}

\begin{abstract}
Robotic cardiac catheters have the potential to revolutionize heart surgery by extending minimally invasive techniques to complex surgical repairs inside the heart. However, catheter technologies are currently unable to track fast tissue motion, which is required to perform delicate procedures inside a beating heart. This paper presents an actuated catheter tool that compensates for the motion of heart structures like the mitral valve apparatus by servoing a catheter guidewire inside a flexible sheath. We examine design and operation parameters and establish that friction and backlash limit the tracking performance of the catheter system. Based on the results of these experiments, we implement compensation methods to improve trajectory tracking. The catheter system is then integrated with an ultrasound-based visual servoing system to enable fast tissue tracking. In vivo tests show RMS tracking errors of $0.77 \mathrm{~mm}$ for following the porcine mitral valve annulus trajectory. The results demonstrate that an ultrasound-guided robotic catheter system can accurately track the fast motion of the mitral valve.
\end{abstract}

Keywords: Catheter, motion compensation, heart, ultrasound, visual servoing

\section{Introduction}

Innovations in catheter technology have greatly expanded the range of procedures that interventional cardiologists can perform inside the heart using minimally invasive techniques. Procedures that are now performed using catheters include measuring cardiac physiological function, dilating vessels and valves, and implanting prosthetics and devices [1]. Nonetheless, catheters do not yet allow clinicians to interact with heart tissue with the same level of skill as in open heart surgery. A primary reason for this deficiency is that current catheters do not have the dexterity, speed, and force capabilities to perform complex or delicate tissue interactions.

Cardiac catheters are long and thin flexible tubes that are inserted into the vascular system and passed into the heart. Current robotic cardiac catheters, such as the commercially available Artisan Control Catheter (Hansen Medical, Mountain View CA, USA), permit a human operator to control the positioning of a catheter in the lateral direction and advance it through the vasculature [2]-[4]. However, these systems do not provide sufficient speeds to compensate for the motion of the heart. Fast motion compensation is required for many beating heart procedures to enable 


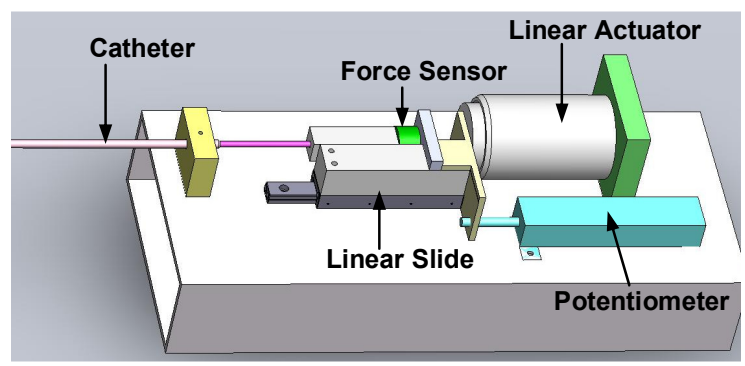

Fig. 1. Actuated catheter system prototype.

dexterous interaction and prevent the catheter from colliding with internal cardiac structures [5].

Researchers have developed robotic approaches to compensating for the motion of the beating heart [6]-[8], but these techniques are directed at coronary artery bypass procedures that repair arteries on the external heart surface. In previous work, we developed robotic devices that compensate for the motion of internal heart structures with a handheld robotic instrument inserted through incisions in the heart wall [5], [9]-[11]. This work shows that single degree of freedom (DOF) servoing is sufficient to accurately track the motion of certain cardiac structures, including the human mitral valve annulus [9],[11]. This approach alleviates the risks associated with stopped heart techniques [12], but the necessity of creating incisions in the heart wall means that this approach is not minimally invasive.

This paper explores the viability of applying our successful robotic cardiac motion compensation techniques to catheters in order to minimize invasiveness. In the envisioned clinical system, an actuator at the base of the catheter system will drive a catheter guidewire inside a flexible sheath (Fig. 1). The sheath is manually advanced through the vasculature into the heart. A standard 3D ultrasound (3DUS) probe images the catheter tip and the tissue target, and real-time image processing algorithms track the catheter-tissue relationship. The guidewire tip is then translated in and out of the sheath to compensate for the cardiac motion and to perform repairs.

The paper begins with a description of the prototype actuated catheter system. Operation of this system reveals a number of challenges that result from quickly translating a guidewire inside a plastic sheath, particularly friction and backlash. This results in position hysteresis and significant tip trajectory errors. We characterize the relationship between catheter design parameters and performance. The insights from these experiments are then used to improve the catheter system tracking through mechanical design and compensation control. Finally, the catheter system is integrated with the ultrasound-based visual servoing system and evaluated with in vivo animal experiments. The results of these experiments demonstrate the feasibility of using catheters for beating-heart intracardiac repair. 


\section{System Design}

The design parameters for the actuated catheter system were selected from the human mitral valve physiology values determined for our earlier handheld motion compensation instrument [5], [11]. The system's principal functional requirements are that it has a single actuated linear degree of freedom with at least $20 \mathrm{~mm}$ of travel that can provide a maximum velocity and acceleration of at least $210 \mathrm{~mm} / \mathrm{s}$ and $3800 \mathrm{~mm} / \mathrm{s}^{2}$, respectively.

The experimental system used in this study (Fig. 1) is composed of a linear voice coil actuator with $50.8 \mathrm{~mm}$ of travel and a peak force of $26.7 \mathrm{~N}$ (NCC20-18-02-1X, H2W Technologies Inc, Valencia CA, USA), a linear ball bearing slide, and a linear potentiometer position sensor. The catheter sheaths are $85 \mathrm{~cm}$ long sections of PTFE (Teflon) tubing, and the guidewires are stainless steel close-wound springs. The geometry of the various combinations of sheaths and guidewires is detailed below. The catheter sheath and guidewire can be flexed as required by the vascular geometry (bent, twisted, etc.) while the guidewire is servoed by the base module.

A PID control system running at $1 \mathrm{kHz}$ is used to control the position of the linear actuator at the base of the catheter. Commands to the linear actuator are amplified by a bipolar voltage-to-current power supply (BOP 36-12M, Kepco Inc., Flushing NY). The reaction forces between the guidewire and actuation mechanism generated by friction and forces applied to the tip of the guidewire are measured with a miniature force sensor (LCFD-1KG, Omega Engineering, Stamford CT, range: $10 \mathrm{~N}$, accuracy: $\pm 0.015 \mathrm{~N})$. In the characterization tests that follow, the catheter tip position is measured with an ultra-low friction rotary potentiometer (CP-2UTX, Midori America Corp, Fullerton CA) connected to the catheter tip with a lightweight lever arm that converts the linear motion into rotation. For the subsequent in vivo studies, tip position is measured with an electromagnetic tracker and ultrasound imaging.

\section{Performance Limitations}

Operation of this prototype system reveals two principal performance limitations: the friction forces experienced by the guidewire, and the backlash behavior due to the guidewire-sheath interaction. These two issues degrade the trajectory tracking accuracy and response time of the actuated catheter end effector. Fig. 2 illustrates an example of the catheter tip failing to accurately track a desired trajectory. A large number of factors are involved in determining the friction and backlash properties of the catheter system. To understand how to best design and control this system, the experimental variables examined in this study include the gap size between the sheath and guidewire and the bending configuration of the catheter system, characterized by the bend radii and bend angles of the catheter sheath (Fig. 4).The catheter material properties and the external forces were held constant. 


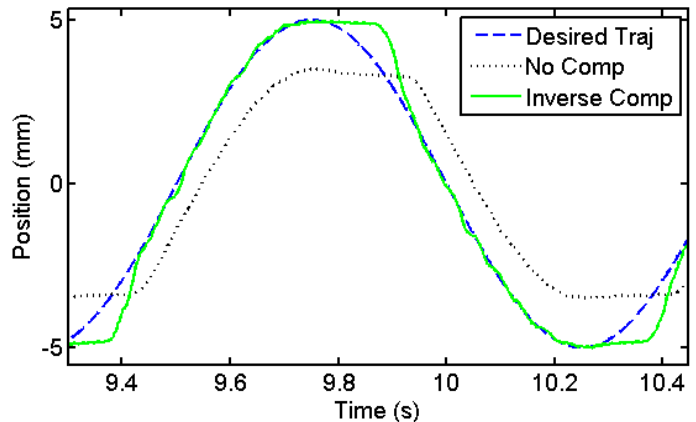

Fig. 2. A sinusoidal desired trajectory and the measured catheter tip trajectory with and without compensation.

\subsection{Friction}

Experimental Methods. The first set of experiments examined the friction of the catheter system as a function of four different sheath-guidewire gap sizes (Table 1), three bending angles $\left(90^{\circ}, 180^{\circ}\right.$, and $\left.360^{\circ}\right)$, and two bend radii $(25$ and $50 \mathrm{~mm})$. The friction was calculated by commanding a series of constant velocities from the actuator in both the positive and negative directions. Force sensor readings during the constant velocity portion of the trajectory were averaged.

Friction Results. Fig. 4 presents a typical friction-velocity curve for this system. The observed behavior can be approximately described as constant dynamic Coulomb friction plus a component that varies linearly with velocity. For this case, the Coulomb term can be approximated as $1.0 \mathrm{~N}$ of friction and the velocity dependent term as $0.006 \mathrm{~N} /(\mathrm{mm} / \mathrm{s})$.

The results of the friction experiments, summarized in Fig. 5, illustrate a number of trends. The data was analyzed with a three-way analysis of variance (ANOVA). The most significant trend is that the gap size has the strongest influence on guidewire friction $(p<0.0001)$. The gap size, i.e. the interior space between the guidewire and the inner wall of the sheath (Fig. 3), directly affects the normal forces applied to the guidewire by the sheath. The normal force is created by any sections of the sheath that might be pinched or kinked, locations where the catheter bending forces the guidewire

TABLE I

\begin{tabular}{ccrc}
\multicolumn{4}{c}{ EXPERIMENTAL CATHETER DIMENSIONS } \\
\hline \hline Symbol & $\begin{array}{c}\text { Sheath Inner } \\
\text { Diameter }\end{array}$ & $\begin{array}{c}\text { Guidewire } \\
\text { Diameter }\end{array}$ & $\begin{array}{c}\text { Gap Size } \\
(G)\end{array}$ \\
\hline$\nabla$ & $1.6 \mathrm{~mm}$ & $0.8 \mathrm{~mm}$ & $0.8 \mathrm{~mm}$ \\
$*$ & $1.6 \mathrm{~mm}$ & $1.5 \mathrm{~mm}$ & $0.1 \mathrm{~mm}$ \\
& $2.4 \mathrm{~mm}$ & $1.5 \mathrm{~mm}$ & $0.9 \mathrm{~mm}$ \\
$\square$ & $2.4 \mathrm{~mm}$ & $2.2 \mathrm{~mm}$ & $0.2 \mathrm{~mm}$ \\
\hline \hline
\end{tabular}

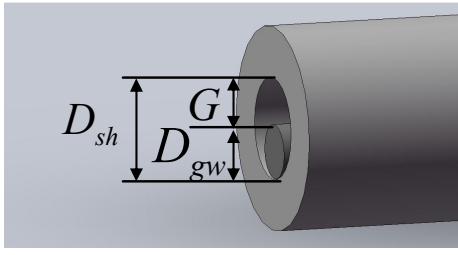

Fig. 3. Sheath and guidewire. 


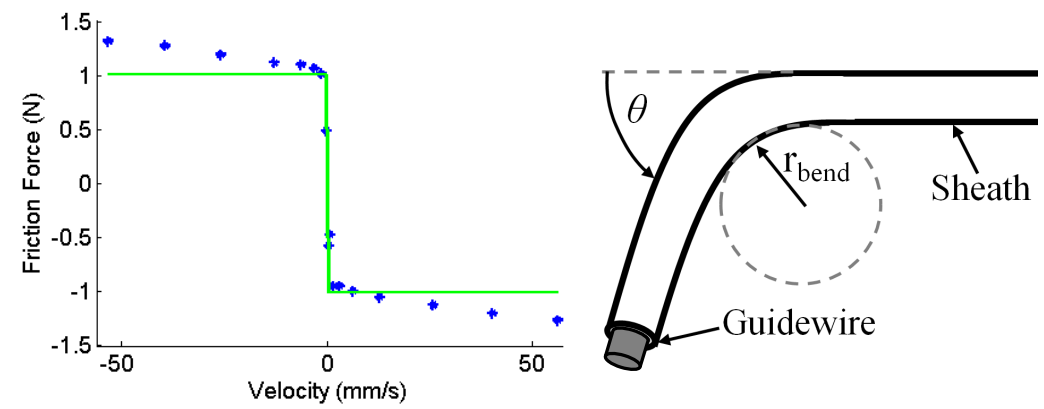

Fig. 4. Left: Friction force versus velocity for the catheter system. The points are experimental values and the line is a Coulombic model approximation of the data. Right: The bending configuration is specified by the bend angle $(\theta)$ and bend radius ( $\mathrm{r}_{\text {bend }}$ ).

to conform along the inner wall of the sheath, and discontinuities in either the guidewire or the sheath that cause the two components to come into contact. The small gap size amplifies these issues because smaller deformations in the catheter system cause the sheath and guidewire to interact. Therefore, increasing the gap size should decrease the friction forces experienced by the guidewire.

The results also show that bend angle has an effect on the friction forces $(p=0.004)$. One reason for this trend is that bending causes the sheaths' cross sections to deform slightly. This deformation can pinch the guidewire, thus increasing the applied normal forces. Also, the bending of the sheath forces the inner guidewire to bend. The reaction forces generated by the conforming guidewire increase the normal force and therefore the friction on the guidewire.

The bending radius does not appear to have a significant impact on the friction measurements $(p=0.64)$, however only two radii $(25 \mathrm{~mm}$ and $50 \mathrm{~mm})$ were examined. These radii were selected because they are approximately the bend radii required to maneuver into the heart.

\subsection{Backlash}

Experimental Methods. The backlash properties of the sheath-guidewire system were investigated with the same experimental variables (gap size, bend angle, bend radius) as the friction experiments present above. The backlash was examined by commanding the base of the catheter system to follow a $1 \mathrm{~Hz}$ sinusoidal trajectory with an amplitude of $5 \mathrm{~mm}$. This trajectory is a highly simplified version of a mitral valve annulus motion of a heart beating at 60 beats per minute (BPM).

The amount of backlash was quantified for each experiment as the width of the backlash hysteresis curve, determined by plotting the commanded trajectory versus the tip position trajectory. The width of the hysteresis is the amount of displacement commanded at the catheter base that does not result in movement at the tip. This backlash width is clearly seen when the commanded trajectory changes directions. 

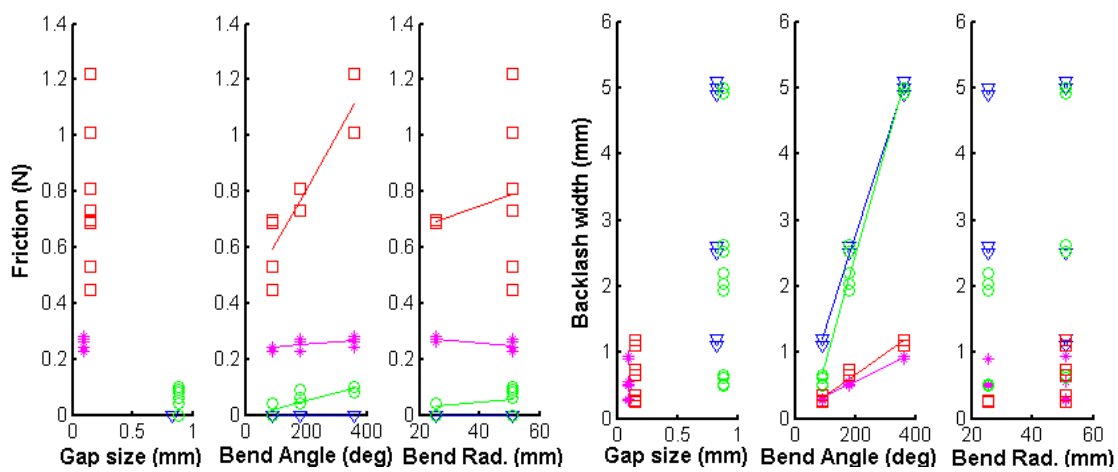

Fig. 5. Summary of the friction results (left) and backlash results (right) versus tested parameters. See Table I for symbols.

Backlash Results. The backlash data (Fig. 5) was analyzed with a three-way ANOVA. Bend angle has the clearest effect on backlash $(p<0.0001)$. The backlash width was proportional to the bend angle. The other parameter that was found to affect the backlash was the gap size $(p<0.0001)$. While the gap size value did not proportionally relate to backlash, the data does suggest that the larger the gap size, the larger the possible amount of backlash. Bend radius was not found to have a systematic effect on the backlash width $(p=0.53)$.

\subsection{Compensation Techniques}

The above results identify the major factors that affect catheter system trajectory tracking performance. This understanding can be used to improve performance through both mechanical design and control system modifications. Friction in the catheter system can be reduced through material selection, material coatings, and lubrication. Backlash can be decreased by reducing the gap between the guidewire and the sheath. However, reducing the gap will also increase the friction experienced by the guidewire.

Control compensation techniques can also reduce friction and backlash effects. For example, feedforward Coulomb friction compensation can be used to reduce the friction forces at the base module [18]. An enhanced control system can also reduce the backlash behavior by modifying the trajectory commanded at the base of the catheter. An example of a standard backlash deadzone compensating method is to solve for the inverse of the backlash [16]. This inverse compensation method is found by adding the system's trajectory tracking error to the original desired trajectory. The addition of the backlash position error term compensates for the deadzone behavior in the system and produces the originally desired trajectory at the output. This method, however, assumes the system can traverse the deadzone instantaneously and that the backlash width is constant and velocity-independent [16]. Fig. 2 presents an example of how inverse compensation can improve the catheter tip trajectory tracking. The 


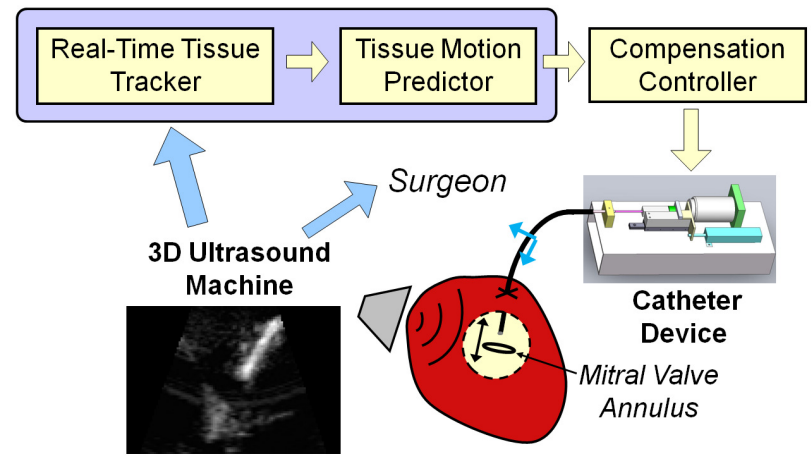

Fig. 6. Ultrasound image-based catheter servoing system.

use of inverse compensation improves the system tracking performance by reducing the mean absolute error (MAE) by $80 \%$.

The results of the friction and backlash experiments, as well as further tests on more complex trajectories, show that backlash imposes more severe performance limitations than friction. In particular, each tip direction reversal requires that the base actuator traverses the entire deadzone in as short a time as possible (e.g. 9.8$9.9 \mathrm{sec}$ in Fig. 2). Actuator force limitations mean this traverse takes long enough for significant errors to develop. Friction compensation, in contrast, can be accomplished to first order by feeding forward the estimated friction. While this changes sign at each direction reversal, the actuator bandwidth for rapid force changes is adequate to avoid substantial errors. This design tradeoff leads us to select a small gap size for subsequent experiments to prevent significant backlash. A full investigation of compensation techniques will be explored in future research.

\section{Image-Based Catheter Control}

To investigate the feasibility of image-based catheter control, we integrated the catheter system described above with the ultrasound visual servoing system we developed in previous work [5], [9]-[11] and evaluated it in vivo. Controlling a catheter to follow the motion of internal cardiac structures requires real-time sensing of both the catheter tip and tissue target positions. 3D ultrasound must be used for guidance because it is currently the only real-time volumetric imaging technique that can image tissue through blood. In our original image guidance system, the tip of a hand-held instrument with a rigid shaft was introduced through a small incision in the heart wall. The instrument successfully tracked the tissue and in vivo experiments demonstrated its ability to lower interaction forces and place anchors in the mitral valve annulus. The ability to perform such tasks with a catheter would enable beatingheart intracardiac repairs to become minimally invasive.

In the ultrasound servoing system, 3D image volumes are streamed via ethernet to an image processing computer (Fig. 6). A GPU-based Radon transform algorithm finds the instrument axis in real-time [10]. The target tissue is then located by 


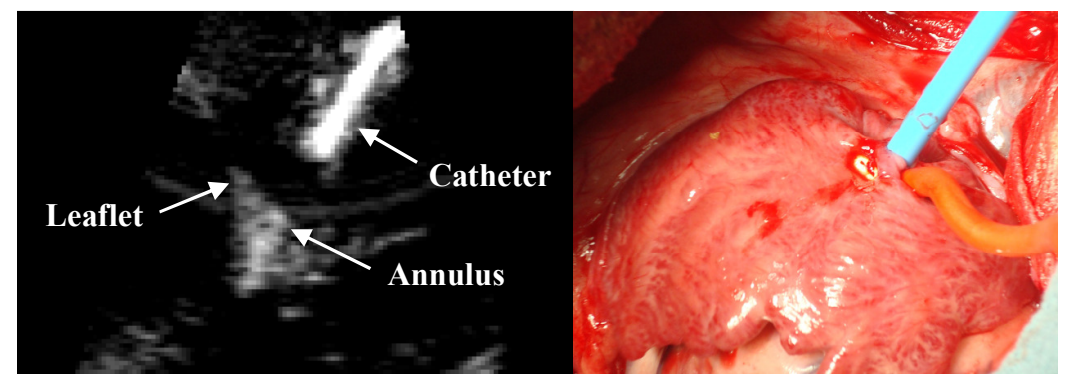

Fig. 7. Left: Ultrasound image showing catheter, mitral valve annulus, and mitral valve leaflets. Right: The catheter tool inserted into the left atrium.

projecting the axis forward through the image volume until tissue is encountered; this allows the clinician to designate the target to be tracked by simply pointing at it with the catheter. To compensate for the $50-100 \mathrm{~ms}$ delay in image acquisition and processing, an extended Kalman filter estimates the current tissue location based on a Fourier decomposition of the cardiac cycle. Previous in vivo experiments showed that the rigid instrument system was capable of accurate tracking within the heart with an RMS error of $1.0 \mathrm{~mm}$. See [9]-[11] for a detailed description of the system.

\subsection{In Vivo Evaluation}

The image guidance system was evaluated in vivo on a $75 \mathrm{Kg}$ porcine animal model. For this initial study, the actuated catheter was inserted into a beating heart via the top of the left atrium rather than the vasculature to give the surgeon easy access to the mitral valve. The 3D ultrasound machine probe was placed epicardially (SONOS 7500, Philips Healthcare, Andover, MA, USA). After the device was introduced into the beating heart, the surgeon used the ultrasound image to aim the catheter at the mitral valve annulus. The imaging system was then initialized and tracked the valve motion. See Fig. 7 for a 3DUS image of the catheter in vivo and an image of the catheter device being inserted into the porcine left atrium.

The catheter system used in this experiment was as described above, with a sheath with $1.6 \mathrm{~mm}$ inner diameter and a guidewire with a $1.5 \mathrm{~mm}$ outer diameter. An electromagnetic tracker (trakSTAR $1.5 \mathrm{~mm}$ sensor, Ascension Technology Corp., Burlington, VT, USA, measured RMS error of $0.3 \mathrm{~mm}$ ) was affixed to the guidewire tip to assess control accuracy.

During the experimental trials, the catheter was fixed external to the heart in a shape with two $90^{\circ}$ bends that roughly corresponds to the path from the femoral vein into the left atrium. The catheter was then positioned inside the left atrium so that the tip was $1-2 \mathrm{~cm}$ from mitral annulus. The catheter controller performs a simple calibration routine that estimates the magnitude of the friction force in the system. Next, the image processing routines locate the catheter using the Radon transform algorithm, and then project forward to find the target. The catheter is then servoed to maintain a constant distance between the catheter tip and the target. 


\subsection{Tracking results}

The catheter system was successful in tracking the mitral annulus tissue target. Fig. 7 shows a cross section through a typical ultrasound image volume, showing the catheter, mitral valve annulus, and edge of the valve leaflet. Friction compensation was used in this experiment, however deadzone compensation was not required because the mechanical design of the catheter system, including the selection of a guidewire and sheath with a small gap size, minimized the deadzone.

The catheter system was tested a number of times during this experiment. Fig. 8 shows a typical plot of the catheter tip trajectory and the position of the mitral valve annulus. This plot was generated by manually segmenting the position of the catheter tip and valve structure from the 3DUS volumes three times and then averaging the values. The standard deviations of all of the segmented tip positions from the mean tip positions were less than $0.22 \mathrm{~mm}$ and the standard deviation of all of the segmented mitral valve annulus positions were less that $0.32 \mathrm{~mm}$ from the mean mitral valve annulus positions. Because of the seals required to prevent backflow of blood out of the heart, friction compensation values as high as $2 \mathrm{~N}$ were required for these experiments

The image guided catheter system tracked the valve motion with RMS errors less that $1.0 \mathrm{~mm}$ in all experimental trials. The RMS error for the trial presented in Fig. 8 was $0.77 \mathrm{~mm}$. The tracking error, shown in Fig. 8, was caused by respiration motion not captured in the tissue tracking system, performance limitations of the actuated catheter caused by backlash and friction, and the small beat-to-beat variations in the valve motion not compensated for by the image tracking system.

\section{Discussion}

Robotic catheters have the potential to revolutionize intracardiac procedures by allowing clinicians to minimally invasively perform complicated surgical tasks inside the beating heart. One of the major technological challenges to realizing this concept is to compensate for the fast motion of cardiac tissue using a catheter. In this paper, we explored the mechanical challenges of servoing a guidewire inside a catheter sheath. Friction increased as a function of bending angle but decreased as a function of the gap size between the guidewire and the sheath. The size of the backlash deadzone was dependent on the gap size and the bending angle. Experiments showed that, compared to friction, backlash creates greater performance deficits and controller compensation is less successful. We therefore selected a small gap size that minimizes backlash at the expense of higher friction.

To investigate the feasibility of using image-based servoing to match catheter trajectories to the motion of cardiac structures, the catheter system was integrated with ultrasound imaging and an image processing system. Porcine in vivo studies showed that excellent tracking can be obtained, with RMS errors of less than one mm. These results are encouraging for the feasibility of this system performing more complicated surgical procedures in vivo. 

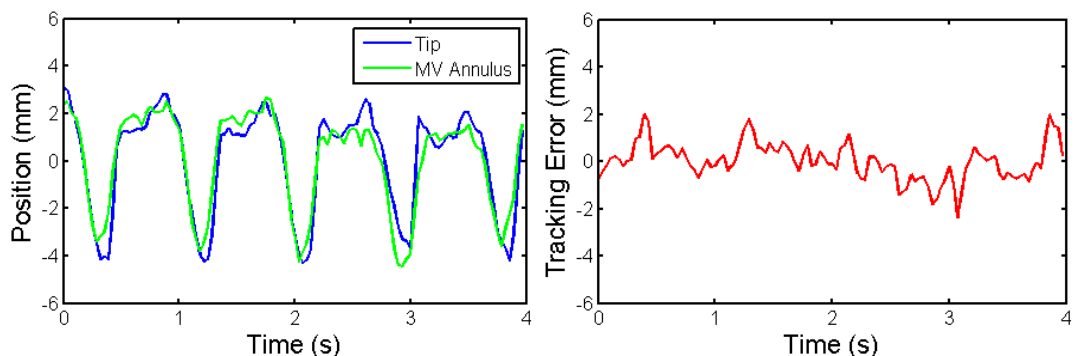

Fig. 8. Left: Trajectory of the catheter tip and the mitral valve annulus found by manual segmentation. Right: The catheter trajectory tracking error.

The next step for this project will be to design adaptive controllers to improve the catheter tip tracking performance in vivo, as well as control the forces applied by the catheter to the cardiac tissue. Optimization of catheter materials and dimensions will be an important aspect of this effort. In addition, it will be essential to determine the durability of catheters under high guidewire velocities and repeated cycling. We note, however, that only a few thousand cycles are needed for a one-hour procedure, and we have operated catheters for this duration with negligible performance degradation.

To the authors' knowledge, the system described here is the first robotic catheter device that can compensate for the fast motion of structures inside the heart. It is interesting to note that this approach is complementary to current commercial catheter robot systems like the Artisan Control Catheter (Hansen Medical, Mountain View CA). That system achieves lateral deflection and sheath translation at roughly manual speeds and could be readily combined with the fast guidewire actuation system described here.

Future work will be required to extend this motion compensation technology to cardiac surgery applications that require additional DOF for end effector positioning and complex tissue trajectory tracking. In contrast to existing robotic catheter systems, fast motion in the lateral directions at approximately the same speeds as demonstrated in this study will be required for these applications.

\section{Conclusion}

This work demonstrates that single DOF robotic catheters can achieve the speed and tip position control required for specific intracardiac repair applications such as mitral valve annuloplasty. In addition, this study shows that catheter position can be accurately controlled using real-time image guidance in vivo. These results suggest that it is feasible to use catheters for beating heart procedures, which will enable intracardiac repairs that are both minimally invasive and avoid the risks of stoppedheart techniques. 


\section{Acknowledgments}

The authors would like to thank Dr. Nikolay Vasilyev and Dr. Pedro del Nido for their assistance with the animal experiments presented here and insights into the clinical applications of this technology. Funding for this work was provided by the US National Institutes of Health under grant NIH R01 HL073647.

\section{References}

[1] D. S. Baim, Grossman's Cardiac Catheterization, Angiography, and Intervention. Lippincott Williams \& Wilkins, 2005, pp. 992.

[2] T. Fukuda, et al., "Micro active catheter system with multi degrees of freedom," in Proc. IEEE Int. Conf. Robotics and Automation, 1994, pp. 2290-2295.

[3] J. Jayender, R. V. Patel and S. Nikumb, "Robot-assisted catheter insertion using hybrid impedance control," in Proc. IEEE Int. Conf. Robotics and Automation, 2006, pp. 607-612.

[4] D. B. Camarillo, C. F. Milne, C. R. Carlson, M. R. Zinn and J. K. Salisbury, "Mechanics Modeling of Tendon-Driven Continuum Manipulators," IEEE Trans. Robotics, vol. 24, pp. 1262-1273, 2008.

[5] S. G. Yuen, S. B. Kesner, N. V. Vasilyev, P. J. del Nido and R. D. Howe, "3D ultrasound-guided motion compensation system for beating heart mitral valve repair," in Medical Image Computing and Computer-Assisted Intervention, LCNS vol. 5241, 2008, pp. 711-719.

[6] O. Bebek and M. Cavusoglu, "Intelligent control algorithms for robotic assisted beating heart surgery," IEEE Trans. Robotics, vol. 23, pp. 468-480, 2007.

[7] R. Ginhoux, et al., "Active filtering of physiological motion in robotized surgery using predictive control," IEEE Trans. Robotics, vol. 21, pp. 67-79, 2005.

[8] Y. Nakamura, K. Kishi and H. Kawakami, "Heartbeat synchronization for robotic cardiac surgery," in Proc. IEEE Int. Conf. Robotics and Automation, 2001, pp. 2014-2019.

[9] S. G. Yuen, P. M. Novotny and R. D. Howe, "Quasiperiodic predictive filtering for robot-assisted beating heart surgery," in Proc. IEEE Int. Conf. Robotics and Automation, 2008, pp. 3875-80.

[10] P. M. Novotny, et al., "GPU based real-time instrument tracking with three-dimensional ultrasound," Medical Image Analysis, 2007, pp. 458-464.

[11] S. G. Yuen, et al., Robotic "Robotic Motion Compensation for Beating Heart Intracardiac Surgery," Int. J. Robotics Research, vol. 28, no. 10, pp. 1355-72, 2009.

[12] M. F. Newman, et al., "Longitudinal Assessment of Neurocognitive Function after Coronary-Artery Bypass Surgery," New England J Med, vol. 344, no. 6, pp. 395-402, 2001.

[13] M. Kaneko, T. Yamashita and K. Tanie, "Basic considerations on transmission characteristics for tendon drive robots," in Proc. Int. Conf. on Advanced Robotics, 1991, pp. 827-832.

[14] A. Nahvi, J. M. Hollerbach, Y. Xu and I. W. Hunter, "An investigation of the transmission system of a tendon driven robot hand," in Proc. IEEE/RSJ Int. Conf. Intelligent Robots and Systems, 1994, pp. 202-208.

[15] G. Palli and C. Melchiorri, "Model and control of tendon-sheath transmission systems," in Proc. IEEE Int. Conf. Robotics and Automation, 2006, pp. 988-993.

[16] M. Nordin and P. Gutman, "Controlling mechanical systems with backlash - a survey," Automatica, vol. 38, pp. 1633-1649, 2002.

[17] E. K. Bassett, A. H. Slocum, P. T. Maslakos, H. I. Pryor, O. C. Farokhzad and J. M. Karp, "Design of a mechanical clutch-based needle-insertion device," PNAS, vol. 106, pp. 5540-5545, 2009.

[18] B. Armstrong-Helouvry, P. E. Dupont and C. Canudas De Wit, "A survey of analysis tools and compensation methods for control of machines with friction," Automatica, vol. 30, pp. 1083-1138, 1994.

[19] D. A. Recker, P. V. Kokotovic, D. Rhode and J. Winkelman, "Adaptive nonlinear control of systems containing a deadzone," in Proc. IEEE Conf. on Decision and Control, 1991, pp. 2111-2115.

[20] R. Horowitz, "Learning Control of Robot Manipulators," Trans. of ASME, vol. 115, pp. 402-411, 1993. 